\title{
Review: donepezil, metrifonate, rivastigmine, and Ginkgo biloba are more effective than placebo in Alzheimer's disease
}

Wolfson C, Moride Y, Perrault A, et al. Drug treatments for Alzheimer's disease. I. A comparative analysis of clinical trials.
Ottawa (ON): Canadian Coordinating Office for Health Technology Assessment (CCOHTA), 2000 May.

\section{QUESTION: Which drug treatments are most effective for adults with Alzheimer's disease (AD)?}

\section{Data sources}

Studies were identified by searching Medline, CINAHL, Applied Science and Technology, Core Biomedical Collection, Core Biomedical Collection III, PsycINFO, HealthSTAR, Cochrane Library, references of review articles, and personal files.

\section{Study selection}

Randomised controlled trials (RCTs) were selected if they were full reports published from 1986 to 1999 on drugs that were on the market or in phase III clinical trials; had a quality score $\geqslant 5$ on the 8 item Jadad scale (maximum score 8); and used NINCDS/ADRDA criteria to diagnose $\mathrm{AD}$.

\section{Data extraction}

Data were extracted on methods and duration of RCT, number of patients, type of drug, completion rates, adverse effects, and outcomes.

\section{Main results}

26 RCTs met the selection criteria. Donepezil: 4 RCTs compared donepezil with placebo for 12 weeks (2 RCTs) or 24 weeks (2 RCTs). A benefit on the Alzheimer Disease Assessment-cognitive subscale (ADAS-cog) was seen for donepezil, $5 \mathrm{mg}$ /day in 4 RCTs (difference in mean change from baseline scores 1.5 to 3.2 ); $10 \mathrm{mg}$ /day in 3 RCTs (difference in mean change from baseline scores 2.9 to 3.1 ); and $3 \mathrm{mg}$ /day in 1 RCT (difference in mean change from baseline scores 2.1). Metrifonate: 6 RCTs compared metrifonate with placebo. All studies had a 2 week screening period, and the double blind phase ranged from $6-26$ weeks. All but 1 RCT had a 2 phase dosing strategy with a loading dose followed by maintenance doses to achieve a predetermined minimum level of acetylcholinesterase inhibition (30-70\%). All studies reported a benefit for metrifonate over placebo on the ADAS-cog. The difference in mean change scores from baseline to end of treatment in 5 RCTs ranged from 1.5 (95\% CI 0.2 to 2.8 ) to 2.9 (CI 1.6 to 4.3). Rivastigmine: 2 RCTs compared low dose (1-4 mg/d) and high dose (6-12 $\mathrm{mg} / \mathrm{d}$ ) rivastigmine with placebo for 26 weeks. 1 RCT $(n=699)$ showed a benefit for both rivastigmine groups in cognition and global functioning (mean score difference in decline on the ADAS-cog 3.8, 95\% CI 2.6 to 4.9 for high dose rivastigmine). In the other RCT $(\mathrm{n}=725)$, a benefit was seen for the high dose group (difference in mean change from baseline scores on the ADAS-cog 1.6) but not for the low dose group. Ginkgo biloba: 3 RCTs compared $G$ biloba with placebo for 12 weeks $(n=20), 24$ weeks $(n=222)$, and 1 year $(n=309)$. Doses were $240 \mathrm{mg} /$ day in $2 \mathrm{RCTs}$ and $120 \mathrm{mg} /$ day in 1 RCT. A benefit for $G$ biloba was seen on the ADAS-cog in 1 RCT (difference in mean change from baseline score 1.7, CI 0.2 to 3.2) and on the Syndrom-Kurz Test in 2
RCTs. Studies did not show a clear benefit in cognitive or global outcomes for propentofylline (2 RCTs), lethicin (1 RCT), linopirdine (1 RCT), vitamin E (1 RCT), or selegiline (7 RCTs).

\section{Conclusion}

In adults with Alzheimer's disease, donepezil, metrifonate, rivastigmine, and Ginkgo biloba are better than placebo for cognitive performance. The data do not show a benefit for vitamin E, lecithin, linopirdine, selegiline, and propentofylline.

\section{COMMENTARY}

Wolfson et al have prepared a comprehensive and rigorous review of the published RCTs of treatments for AD. Differences in methodology, including design and reporting strategies, precluded a meta-analysis. Many of the studies reviewed were designed to meet the research criteria for drug licensing: investigation of efficacy in treatment of $\operatorname{cog}_{-}$ nitive impairment in dementia as well as drug safety. Studies included in the review were of short duration; some studies had potential for bias from high dropout rates and some studies had participants unlike the usual patient with comorbid disorder.

This abstract only reports on the outcome measure of cognitive function. The review itself highlights some of the problems with the measurements of other clinical outcomes. The abstracted treatment effects are differences in points on the ADAS-cog scale (range 0-70). Average treatment effects of cholinesterase inhibitors were $1.5-3.8$ points. Somewhat smaller effects were recorded for G biloba. Although these were statistically significant, clinical importance is not clear Some people respond to treatment better than others. The characteristics of those who respond well, however, are not known.

From these data we can advise patients that some evidence exists to support the short term use of donepezil, metrifonate, and rivastigmine. Clinical discussion should consider the lack of data on longer term use, including the consequences of withdrawing the treatment and its potential for side effects.

We also want to know the implications of these treatments for psychiatric and behavioural aspects of $\mathrm{AD}$ as well as for cognitive ones. The recently published RCT of galantamine provides data on response in all 3 types of symptoms in dementia. ${ }^{1}$ In addition to treatment effects on cognitive function, there may be significant benefits for non-cognitive symptoms. Much of the research so far has been on patients with mild to moderate dementia. An important question therefore is whether these treatments may be effective in more severe dementia.

Jane Pearce, BMed Sci, BM, BS, MRCGP, MRCPsych The Fulbrook Centre Oxford, UK

1 Tariot PN, Solomon PR, Morris JC, et al. A 5-month, randomised, placebo-controlled trial of galantamine in $\mathrm{AD}$. The Galantamine USA-10 Study Group. Neurology 2000;54:2269-76. 